\title{
A NOTE ON SUBORDINATION
}

\author{
SHIGEYOSHI OWA \\ Department of Mathematics \\ Kinki University \\ Osaka, Japan \\ (Received March 20, 1984)
}

ABSTRACT. Suffridge showed a result for subordinate functions. The object of the present paper is to show some subordinate theorems with the aid of the result by Suffridge.

¿'BY WORDS AND PH'ASE'. SUbordinate, Starlike of order $\alpha$, convex of order $\alpha$. l980 AM SURJECT CLASSIFICATTON CODE. 30C45.

1. INTRODUCTION.

Let $f(z)$ and $g(z)$ be analytic in the unit disk $u=\{z:|z|<1\}$. A function $\mathrm{f}(z)$ is said to be subordinate to $\mathrm{g}(z)$ if there exists a function $\phi(z)$ analytic in the unit disk $u$ satisfying $\phi(0)=0$ and $|\phi(z)|<1(z \in U)$ such that $f(z)=$ $g(\phi(z))$ for $z \in U$. We denote by $f(z) \mathcal{L}_{g}(z)$ this relation. In particular, if $g(z)$ is univalent the unit disk $u$ the subordination is equivalent to $f(0)=g(0)$ and range $f(z) \subset$ range $g(z)$.

This concept of subordination can be traced to Lindelof [1], but Littlewood [2],[3] and Rogosinski [4], [5] introduced the term and discovered the basic properties. Recently Suffridge [6] and Hallenbeck and Ruscheweyh [7] studied the subordinate functions and showed many interesting results for subordinations.

Let $A$ dente the class of funrtions of the form

$$
f(z)=z+\sum_{n=2}^{\infty} a_{n} z^{n}
$$

which are analytic in the unit disk $U$. Further let $S$ be the subclass of $A$ consisting of analytic and univalent functions in the unit disk $U$. Then a function $f(z)$ of $S$ is said to be starlike of order $\alpha$ if and only if

$$
\operatorname{Re}\left\{\frac{z f^{\prime}(z)}{f(z)}\right\}>a
$$

for some $\alpha\left(0 \leqq a^{\circ}<1\right)$. We derote by $S^{*}(\alpha)$ the class of all starlike functions of order $\alpha$. Further a function $f(z)$ of $S$ is said to be convex of order $\alpha$ if and only if

$$
\operatorname{Re}\left\{1+\frac{z f^{\prime \prime}(z)}{f^{\prime}(z)} ;:=\alpha \quad(z, u)\right.
$$

for some $\alpha(0 \leq \alpha<1)$. And we denote by $K(\alpha)$ the class of convex functions of 
order $\alpha$. it is well-known that $f(z) \in K(\alpha)$ if and only if $z f^{\prime}(z)<S^{*}(\alpha)$, $S^{*}(\alpha) \subset S^{*}, K(a) \subset K$, and $S^{*}(0) \equiv S^{*}, K(0) \equiv K$ for $\alpha=0$.

The classes $S^{*}(\alpha)$ and $K(\alpha)$ were first introduced by Robertson [8], and latter studied by Schild [9], MacCregor [10] and Pinchuk [11]. Further, recently, some] classes defined by using the extremal function $z /(1-z)^{2(1-\alpha)}$ for $s^{*}(\alpha)$ were studied by Ruscheweyh [12], Sheil-Small, Silverman and Silvia [13], Silverman and Silvia [14], and Ahuja and Silverman [15].

Our main tool in this paper is the following result by Suffridge [15].

LEMMA. Let the function $f(z)=\sum_{n=2}^{\infty} a_{n} z^{n}$ be analytic in the unit disk $u$ and the function $g(z)$ be in the class $S^{*}$. If $f(z)$ is subordinate to $g(z)$, that is, $\mathrm{f}(\mathrm{z})<\mathrm{g}(\mathrm{z})$, then

$$
\int_{0}^{z} \frac{f(t)}{t} d t \propto \int_{0}^{z} \frac{g(t)}{t} d t
$$

for $\mathrm{z} \in \mathrm{u}(\mathrm{r})=\{\mathrm{z}:|\mathrm{z}| \leqq \mathrm{r}, 0 \leqq \mathrm{r}<1\}$.

2. SUBORDINATION THEOREMS.

In this section, we show some subordination theorems with the aid of Lemma.

THEOREM 1. Let the function $f(z)$ defined by (1.1) be in the class of $K(\alpha)$. Then $f^{\prime}\left(r e^{i \theta}\right)(0 \leqq r<1)$ is contained in the image domain of the closed disk u'r) under the function $e^{4(\alpha-1) /(1-z)}$. Further it lies for $r \neq 0$ on the boundary of of this image domain if and only if

$$
f(z)=\int_{0}^{z} e^{4(1-\alpha) /(1-\varepsilon t)} d t
$$

where $|\varepsilon|=1$.

PROOF. Since $f(z)$ is in the class $K(\alpha), f(z)$ satisfies that

$$
\operatorname{Re}\left\{\frac{z f^{\prime \prime}(z)}{f^{\prime}(z)}\right\}>\alpha-1
$$

Note that $z f^{\prime \prime}(z) / f^{\prime}(z)=2 a z+\ldots$ is analytic in the unit disk $u$, and that the function $z /(1-z)^{2}$ is starlike with respect to the orgin and $\operatorname{Re}\left\{z /(1-z)^{2}\right\}>-1 / 4$. Hence we have that

$$
\frac{z f^{\prime \prime}(z)}{f^{\prime}(z)} \prec \frac{4(1-\alpha) z}{(1-z)^{2}}
$$$$
(z \in U)
$$

Consequently, by using Lemma, it follows that $\log \mathrm{f}^{\prime}\left(\mathrm{re}^{i \theta}\right)$ is contained in the image domain of $U(r)$ under the function $4(\alpha-1) /(1-z)$, where $\log$ is understood to be that branch which vanishes at the point one. Thus we can see that $f^{\prime}\left(r e^{i \theta}\right)$ lies for $r \neq 0$ on the boundary of the image domain of $u(r)$ under $e^{4(\alpha-1) /(1-z) \text {. Further }}$ $f^{\prime}\left(r e^{i \theta}\right)$ lies for $r \neq 0$ on the boundary of the image domain $u(r)$ under $e^{4(\alpha-1) /(1-z)}$ if and only if $\frac{z f^{\prime \prime}(z)}{f^{\top}(\because)}=\frac{4(1-\alpha) \varepsilon z}{(1-\varepsilon z)^{2}}$

$$
(|\varepsilon|=1) \text {, }
$$
hence further, $f(z) \quad i$ the funition of the form (2.1). This completes the proof uf the theorem. 
THEOREM 2. Let the function $f(z)$ defined by $(1.1)$ be in the class $s^{*}(\alpha)$. Then $f\left(r e^{i \theta}\right) / r e^{i \theta} \quad(0 \leqq r<1)$ is contained in the image domain of the closed disk $u(r)$ under the function $e^{4(-1) /(1-z)}$. Further it lies for $r \neq 0$ on the boundary of this image domain if and only if $f(z)=z e^{4(1-\alpha) /(1-\varepsilon z)}$, where $|\varepsilon|=1$.

PROOF. Since $f(z) \in S^{*}(\alpha), f(z)$ satisfies that

and the function $z f^{\prime}(z) / f(z)\left\{\frac{z f^{\prime}(z)}{f(z)}-1\right\}>a-1 \quad$ (ztu) $z f^{\prime}(z) / f(z)-1$ takes values in the image domain of the unit disk $u$ under the function $4(1-\alpha) z /(1-z)^{2}$, that is,

$$
\frac{z f^{\prime}(z)}{f(z)}-1<\frac{4(1-\alpha) z}{(1-z)^{2}}
$$

By virtue of Lemma, we observe that $\log \mathrm{f}\left(\mathrm{re}^{i \theta}\right) / \mathrm{re} \mathrm{i \theta}^{i \theta}(0 \leq \mathrm{r}<1)$ is contanined in in the image domain of $u(r)$ under $4(\alpha-1) /(1-z)$ and it lies for $r \neq 0$ on the boundary of this image domain if and only if

$$
\frac{z f^{\prime}(z)}{f(z)}-1=\frac{4(1-\alpha) \varepsilon z}{(1-\varepsilon z)^{2}} \quad(|\varepsilon|=1),
$$

hence further, $f(z)=z e^{4(1-\alpha) /(1-\varepsilon z)}$. This gives the result we require.

Finally we show a theorem for functions $f(z)$ satisfying $\operatorname{Re}\left\{z f^{\prime}(z)\right\}>\alpha(\alpha>0)$.

THEOREM 3. Let the function $f(z)$ defined by $(1.1)$ satisfy $\operatorname{Re}\left\{z f^{\prime}(z)\right\}>\alpha(\alpha>0)$. Then $f\left(r e^{i \theta}\right) \quad(0 \leq r<1)$ is contained in the image domain of the closed disk $u(r)$ under the function $-4 \alpha /(1-z)$. Further it lies for $r \neq 0$ on the boundary of this domain if and only if $f(z)=-4 \alpha /(1-\varepsilon z)$, where $|\varepsilon|=1$.

PROOF. We note that the function $z f^{\prime}(z)=z+2 a_{2} z^{2}+\ldots$ takes values in the image domain of the unit disk $u$ under the function $-4 a z /(1-z)^{2}$ which belongs to the class $S^{*}$. Therefore we can prove the theorem by using the same technique as in the one of Theorem 1 with the aid of Lemma.

\section{REFERENCES}

1. LINDELÖF, E. Mémoire sur certaines inégalités dans la theorie des fonctions monogénes et sur quelques proprietés nouvelles de ces fonctions dans le voisinage d'un point singulier essentiel, Acta Soc. Sci. Fenn. 35 (1909), 1-35.

2. LITTLEWOOD, J.E. On inequalities in the theory of functions, Proc. London Math. Soc. 23 (1925), 481-519.

3. LITTLEWOOD, J.E. Lectures on the theory of functions, Oxford University Press, London, 1944.

4. ROGOSINSKI, W. On subordinate functions, Proc. Cambridge Philos. Soc. 35 (1939), $1-26$.

5. ROGOSINSKI, W. On the coefficients of subordinate functions, Proc. London Math. Soc. $48(1943), 48-82$. 6. SUFFRIDGE, T.J. Some remarks on convex maps of the unit disk, Duke Math. J. 37
$(1970), 775-777$. 
7. HALLENBECK, D.J., and RUSCHEWEYH, St. Subordination by convex functions, Proc. Amer. Math. Soc. 52 (1975), 191-195.

8. ROBERTSON, M.S. On the theory of univalent functions, Ann. Math. 37 (1936), 374408 .

9. SCHILD,A. On starlike functions of order $\alpha$, Amer. J. Math. 87 (1965), 65-70.

10. MacGREGOR, T.H. The radius of convexity for starlike functions of order $1 / 2$, Proc. Amer. Math. Soc. 14 (1963), 71-76.

11. PINCHUK, B. On starlike and convex functions of order $\alpha$, Duke Math. J. 35 (1968), 721-734.

12. RUSCHEWEYH, St. Linear operators between classes of prestarlike functions, Comm. Math. Helv. 52 (1977), 497-509.

13. SHEIL-SMALL, T., SILVERMAN, H. and Silvia, E. Convolution multipliers and starlike functions, J. Analyse Math. 41 (1982), 181-192.

14. SILVERMAN, H. and SILVIA, E. Subclasses of prestarlike functions, (to appear).

15. AHUJA, O.P. and SILVERMAN, H. Convolutions of prestarlike functions, Internat. J. Math. Math. Sci. 6 (1983), 59-68. 


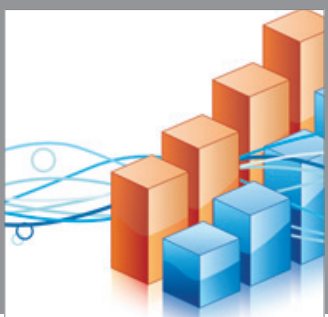

Advances in

Operations Research

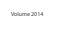

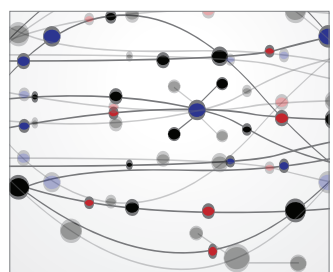

\section{The Scientific} World Journal
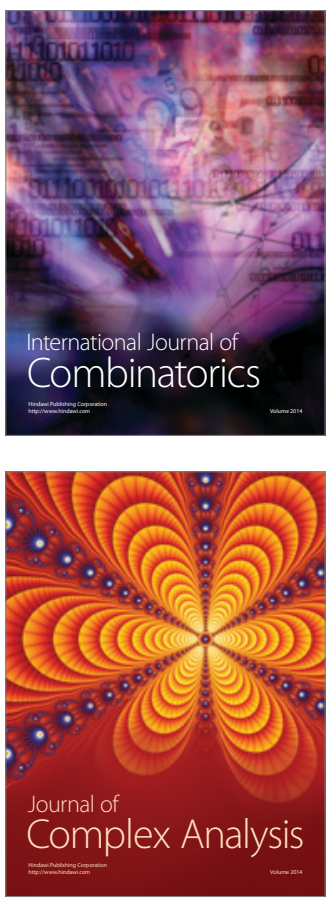

International Journal of

Mathematics and

Mathematical

Sciences
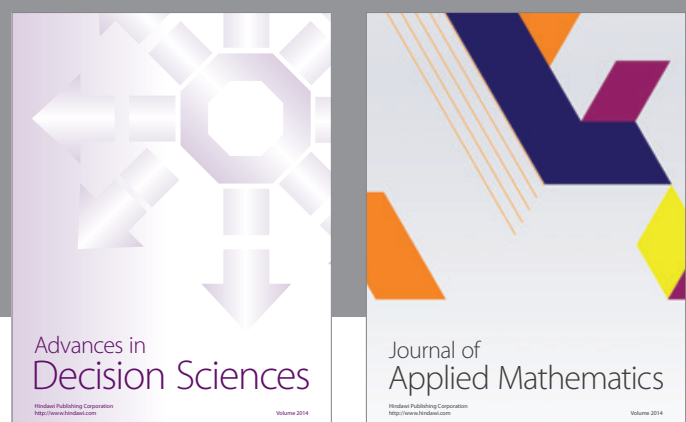

Journal of

Applied Mathematics
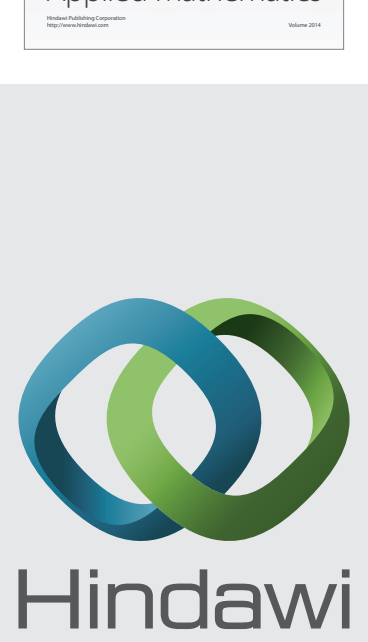

Submit your manuscripts at http://www.hindawi.com
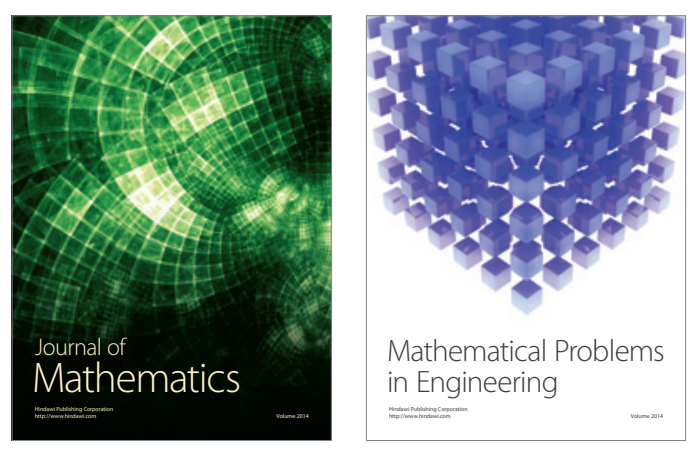

Mathematical Problems in Engineering
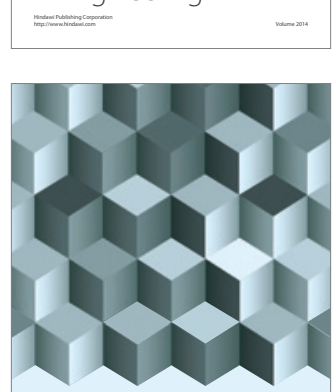

Journal of

Function Spaces
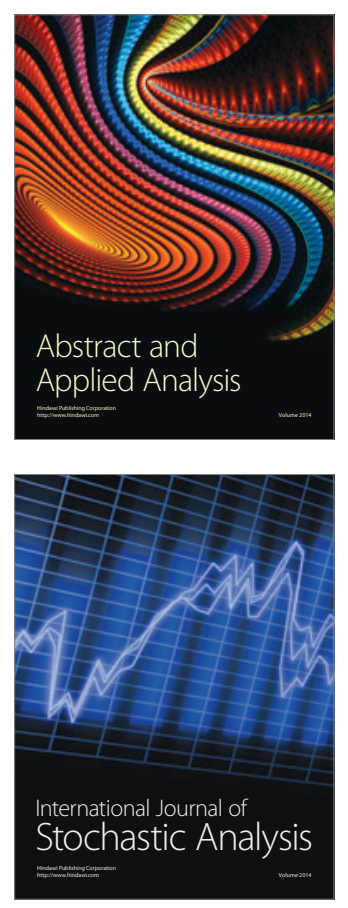

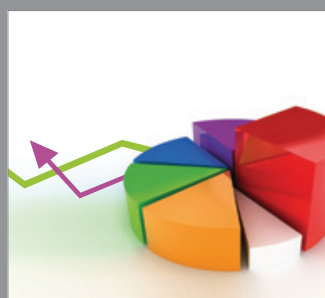

ournal of

Probability and Statistics

Promensencen
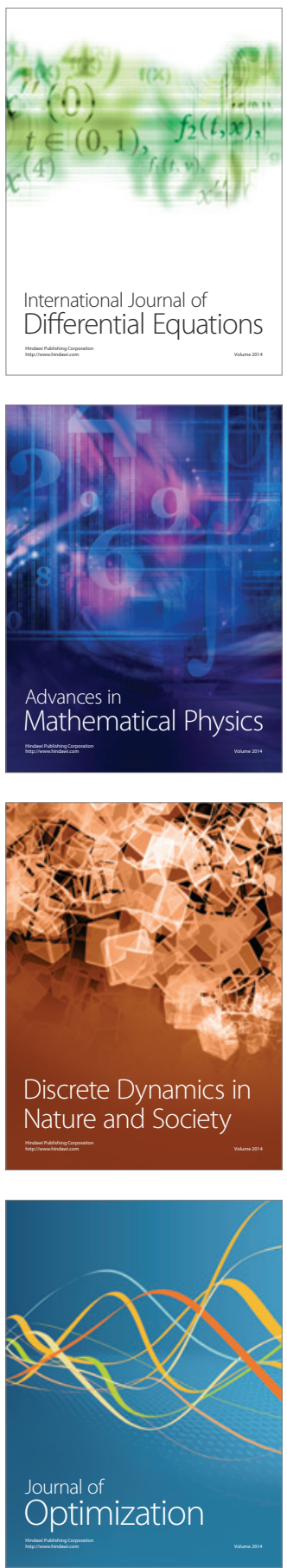